\title{
Scattering from a Buried PEMC Cylinder due to a Line Source Excitation above a Planar Interface between Two Isorefractive Half Spaces
}

\author{
Abdul Kadir Hamid ${ }^{*}$, Francis Cooray ${ }^{2}$ \\ ${ }^{1}$ Department of Electrical and Computer Engineering, University of Sharjah, United Arab Emirates \\ ${ }^{2} 1$ Rowe Street, Eastwood, NSW 2122, Australia \\ *corresponding author, E-mail: akhamidesharjah.ac.ae
}

\begin{abstract}
A solution to the problem of scattering from a perfect electromagnetic conducting (PEMC) circular cylinder buried inside a half-space and excited by an infinite electric line source is provided. The line source is parallel to the cylinder axis, and is located in the other half-space. The two half spaces are isorefractive to each other. The source fields when incident at the planar interface separating the two half spaces, generate fields that are transmitted into the half-space where the cylinder is. These fields then become the known basic incident fields for the buried PEMC cylinder. Scattering of these incidents fields by the cylinder will consequently generate fields at the interface that get reflected back into the same half-space and transmitted frontward into the source half-space, all of which are unknown. Imposing appropriate boundary conditions at the surface of the buried cylinder and at a specified point on the interface, enables the evaluation of these unknown fields. The refection coefficient at the specified point is then computed for cylinders of different sizes, to demonstrate how it varies with the PEMC admittance of the buried cylinder, the intrinsic impedance ratio of the two isorefractive half-spaces, and the burial depth of the cylinder.
\end{abstract}

\section{Introduction}

This work encompasses two media which are divided by a planar interface and isorefractive to each other. Such media have the same wavenumber, but different wave impedances. Thus, the field behaviour in these media is different to that in a regular dielectric medium. Recently, there has been a quite a bit of interest in the open literature, on solutions to problems pertaining to isorefractive media [1-4].

Obtaining solutions to problems concerning buried bodies are valuable, as these may be employed for the detection of underground tunnels, mines, pipes, and other similar objects. Such solutions, using a range of cylindrical wave expressions, have been obtained in the open literature for cylinders which are buried in a homogenous/nonhomogeneous ground, in a lossy earth, and also buried in quite a few rough surfaces [516]. Numerical solutions for scattering from arbitrary shaped buried bodies have been obtained in [17-18] using the Method of Moments (MoM). Scattering by a perfect electromagnetic conductor half-screen is considered in [19].

The objective of this paper is to obtain a rigorous solution to scattering from a PEMC circular cylinder buried in a half- space, when it is excited by a line source infinitely extending parallel to the cylinder axis and placed in the other half-space that is separated by a planar interface, and isorefractive to the half-space in which the cylinder is buried.

In [4], a PEC cylinder located at a planar interface between two isorefractive half-spaces is considered, with the emphasis being on extracting the high frequency asymptotic diffraction contribution from the series solution to the problem, by means of creeping wave theory, when the PEC cylinder is illuminated by a plane wave. In contrast, our work in this paper considers the more general case of a PEMC cylinder buried inside an isorefractive half-space, and excited by an electric line source. Also, unlike in [4], we consider multiple reflections from the interface separating the two half-spaces when formulating the problem, making the formulation quite plain, and not needing the evaluation of any special functions such as Airy functions used in [4].

Scattering of a normally incident plane wave by a PEMC circular cylinder located in free space has been considered in [20]. In contrast, our work considers a PEMC circular cylinder buried within an isorefractive half-space, the excitation being by means of an electric line source, instead of a plane wave, and taking into account multiple reflections from the interface separating the half-spaces. The customary way of considering multiple interactions between the buried circular cylinder and the interface is by employing plane wave representation of the fields using an infinite integral, with the integration being over the wavenumber of the plane wave, making the expressions of the fields quite complex. In our work, the fields are expressed in terms of simple series expressions involving either Bessel or Hankel functions, making the analysis quite simple.

The fields scattered by the buried PEMC cylinder owing to the fields that are incident on it are expressed using a set of wave functions and unknown expansion coefficients. Out of those fields which are generated as a result of these scattered fields being incident on the interface, a portion gets reflected back and the remainder gets transmitted into the source halfspace. They also have expansions similar to those of the fields scattered by the cylinder. The unknown expansion coefficients are evaluated by enforcing appropriate boundary conditions at the surface of the PEMC cylinder and at a specified point on the interface. This is then repeated for multiple reflections and transmissions at the interface and multiple cylinder scatterings until a specific reflection coefficient accuracy is attained at the above specified point on the planar interface. This method is computationally very efficient, as it does not involve inversion 
of matrices to obtain the unknown coefficients. The following sections contain the details of the paper.

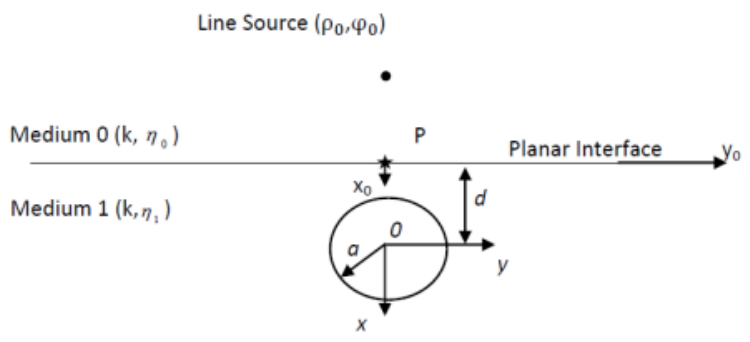

Figure 1: Geometry of the buried PEMC cylinder. The cylinder is located in an isorefractive lower half-space at a distance $d$ from an interface separating the two isorefractive half-spaces, and is excited by a line source located in the upper half-space.

\section{Formulation}

An infinitely long PEMC circular cylinder of radius $a$ is buried in a half-space and positioned at a distance $d$ from a planar interface separating this half-space from another one, as seen in Fig. 1, with the two half-spaces being isorefractive to each other, possessing identical wavenumbers, but different intrinsic wave impedances. An infinite electric line source that extends parallel to the axis of the cylinder is located in the upper half-space at a source point having polar coordinates $\left(\rho_{0}, \phi_{0}\right)$, relative to the Cartesian coordinate system $\left(x_{0}, y_{0}, z_{0}\right)$ in Figure 1, with $\phi_{0}=\pi$.

Components of the incident electric and magnetic fields due to the electric line source can be written using the polar coordinates $(\rho, \phi)$ relative to the Cartesian coordinate system $\left(x_{0}, y_{0}, z_{0}\right)$ in Fig. 1 and the associated vectors $\bar{\rho}$ and $\bar{\rho}_{0}$ as

$$
\begin{aligned}
& E_{z}^{i}=-I H_{0}^{(2)}\left(k\left|\bar{\rho}-\bar{\rho}_{0}\right|\right) \\
& H_{\phi}^{i}=-\frac{I}{j \eta_{0}} H_{0}^{(2) '}\left(k\left|\bar{\rho}-\bar{\rho}_{0}\right|\right)
\end{aligned}
$$

where $k$ is the wavenumber in and $\eta_{0}$ is the wave impedance of the upper half-space, and $I$ is the amplitude of the electric line source. The above incident field components can now be expanded in terms of cylindrical wave functions for $\rho<\rho_{0}$ as

$$
\begin{aligned}
& E_{z}^{i}=-I \sum_{n=-\infty}^{\infty} H_{n}^{(2)}\left(k \rho_{0}\right) J_{n}(k \rho) e^{j n\left(\phi-\phi_{0}\right)} \\
& H_{\phi}^{i}=-\frac{I}{j \eta_{0}} \sum_{n=-\infty}^{\infty} H_{n}^{(2)}\left(k \rho_{0}\right) J_{n}^{\prime}(k \rho) e^{j n\left(\phi-\phi_{0}\right)}
\end{aligned}
$$

where $J_{n}(x)$ is the Bessel function of order $n$ and argument $x$, the prime denoting the derivative of $J_{n}(x)$ with respect to $x$, and $H_{n}{ }^{(2)}(x)$ is the Hankel function of the second kind of order $n$ and argument $x$. The field components of the reflected and transmitted waves generated due to the fields from the electric line source incident on the planar interface (in the absence of the cylinder) can be written as

$$
\begin{aligned}
& E_{z}^{r}=-R I \sum_{n=-\infty}^{\infty} H_{n}^{(2)}\left(k \rho_{0}\right) J_{n}(k \rho) e^{j n\left(\phi+\phi_{0}-\pi\right)} \\
& H_{\phi}^{r}=\frac{R I}{j \eta_{0}} \sum_{n=-\infty}^{\infty} H_{n}^{(2)}\left(k \rho_{0}\right) J_{n}^{\prime}(k \rho) e^{j n\left(\phi+\phi_{0}-\pi\right)} \\
& E_{z}^{t}=-T I \sum_{n=-\infty}^{\infty} H_{n}^{(2)}\left(k \rho_{0}\right) J_{n}(k \rho) e^{j n\left(\phi-\phi_{0}\right)}
\end{aligned}
$$

$$
H_{\phi}^{t}=-\frac{T I}{j \eta_{1}} \sum_{n=-\infty}^{\infty} H_{n}^{(2)}\left(k \rho_{0}\right) J_{n}^{\prime}(k \rho) e^{j n\left(\phi-\phi_{0}\right)}
$$

where $\eta_{1}$ is the wave impedance of the lower half-space, and $R$ and $T$ are the reflection and transmission coefficients owing to the presence of the interface, which are unknown. These can be obtained by imposing the boundary conditions at the planar interface at $x_{0}=0$ as

$$
\begin{array}{r}
R=\frac{\eta_{1}-\eta_{0}}{\eta_{1}+\eta_{0}} \\
T=\frac{2 \eta_{1}}{\eta_{1}+\eta_{0}} .
\end{array}
$$

The initial transmitted electric and magnetic fields linked to their components given by (7) and (8) when incident on the buried PEMC cylinder, will be scattered by it. The co-polar and cross polar components of these scattered fields can be written as

$$
\begin{aligned}
E_{z}^{s, 0} & =\sum_{n=-\infty}^{\infty} B_{n}^{0} H_{n}^{(2)}(k \rho) e^{j n \phi} \\
H_{\phi}^{s, 0} & =\frac{1}{j \eta_{1}} \sum_{n=-\infty}^{\infty} B_{n}^{0} H_{n}^{(2)^{\prime}}(k \rho) e^{j n \phi} \\
H_{z}^{s, 0} & =\frac{1}{j \eta_{1}} \sum_{n=-\infty}^{\infty} C_{n}^{0} H_{n}^{(2)}(k \rho) e^{j n \phi} \\
E_{\phi}^{s, 0} & =\sum_{n=-\infty}^{\infty} C_{n}^{0} H_{n}^{(2)^{\prime}}(k \rho) e^{j n \phi}
\end{aligned}
$$

where $H_{n}^{(2)}(k \rho)$ and $H_{n}^{(2) '}(k \rho)$, respectively, are the second kind of Hankel function and its derivative with respect to the argument, of order $n$ and argument $k \rho$, and $(\rho, \phi)$ are the polar coordinates relative to the Cartesian coordinate system located at the center of the buried cylinder. $B_{n}^{0}$ in (11) - (12) and $C_{n}^{0}$ in (13) - (14) are those undetermined co-polar and cross polar scattered field expansion coefficients, which can be obtained by applying the PEMC boundary condition at the surface of the buried PEMC cylinder. The explicit expressions for these coefficients can be written as [20]

$$
\begin{gathered}
B_{n}^{0}=\frac{H_{n}^{(2)}(k a) J_{n}^{\prime}(k a)+M^{2} \eta_{1}^{2} H_{n}^{(2)^{\prime}}(k a) J_{n}(k a)}{\left(1+M^{2} \eta_{1}^{2}\right) H_{n}^{(2)}(k a) H_{n}^{(2))^{\prime}}(k a)} A_{n} \\
C_{n}^{0}=\frac{2 M \eta_{1}}{\pi k a\left(1+M^{2} \eta_{1}^{2}\right) H_{n}^{(2)}(k a) H_{n}^{(2) '}(k a)} A_{n}
\end{gathered}
$$

where $M$ is the PEMC admittance of the PEMC cylinder, and

$$
A_{n}=T I \sum_{m=-\infty}^{\infty} H_{m}^{(2)}\left(k \rho_{0}\right) J_{m-n}(k d) e^{j m \phi_{0}}
$$

The co-polar $\left(E_{z}^{s, 0}, H_{\phi}^{s, 0}\right)$ and the cross polar $\left(E_{\phi}^{s, 0}, H_{z}^{s, 0}\right)$ components of the scattered field in (11)-(14) incident on the planar interface, generate transmitted co-polar $\left(E_{z}^{t, 0}, H_{\phi}^{t, 0}\right)$ and cross polar $\left(E_{\phi}^{t, 0}, H_{z}^{t, 0}\right)$ field components propagated forward into the upper half-space, as well as reflected copolar $\left(E_{z}^{r, 0}, H_{\phi}^{r, 0}\right)$ and cross polar $\left(E_{\phi}^{r, 0}, H_{z}^{r, 0}\right)$ field components propagated back into the lower half-space as second order field components that are subsequently incident on the buried PEMC circular cylinder. These transmitted field components can be expressed in the form 


$$
\begin{aligned}
E_{z}^{t, 0} & =\sum_{n=-\infty}^{\infty} D_{n}^{0} H_{n}^{(2)}(k \rho) e^{j n \phi} \\
H_{\phi}^{t, 0} & =\frac{1}{j \eta_{0}} \sum_{n=-\infty}^{\infty} D_{n}^{0} H_{n}^{(2)^{\prime}}(k \rho) e^{j n \phi} \\
H_{z}^{t, 0} & =\frac{1}{j \eta_{0}} \sum_{n=-\infty}^{\infty} F_{n}^{0} H_{n}^{(2)}(k \rho) e^{j n \phi} \\
E_{\phi}^{t, 0} & =\sum_{n=-\infty}^{\infty} F_{n}^{0} H_{n}^{(2)^{\prime}}(k \rho) e^{j n \phi}
\end{aligned}
$$

and the reflected field components in the form

$$
\begin{aligned}
E_{z}^{r, 0} & =\sum_{n=-\infty}^{\infty} G_{n}^{0} H_{n}^{(2)}\left(k \rho_{\text {imag }}\right) e^{j n \phi_{\text {imag }}} \\
H_{\phi}^{r, 0} & =\frac{1}{j \eta_{1}} \sum_{n=-\infty}^{\infty} G_{n}^{0} H_{n}^{(2))^{\prime}}\left(k \rho_{\text {imag }}\right) e^{j n \phi_{\text {imag }}} \\
H_{z}^{r, 0} & =\frac{1}{j \eta_{1}} \sum_{n=-\infty}^{\infty} Q_{n}^{0} H_{n}^{(2)}\left(k \rho_{\text {imag }}\right) e^{j n \phi_{\text {imag }}} \\
E_{\phi}^{r, 0} & =\sum_{n=-\infty}^{\infty} Q_{n}^{0} H_{n}^{(2)^{\prime}}\left(k \rho_{\text {imag }}\right) e^{j n \phi_{\text {imag }}}
\end{aligned}
$$

where $\left(\rho_{\text {imag }}, \phi_{\text {imag }}\right)$ are the polar coordinates pertaining to the Cartesian coordinate system located at the center of the image cylinder as shown in Fig. 2, $D_{n}^{0}$ and $G_{n}^{0}$ are the unknown copolar transmitted and the co-polar reflected field coefficients, respectively, and $F_{n}^{0}$ and $Q_{n}^{0}$ are the analogous unknown cross polar field coefficients.

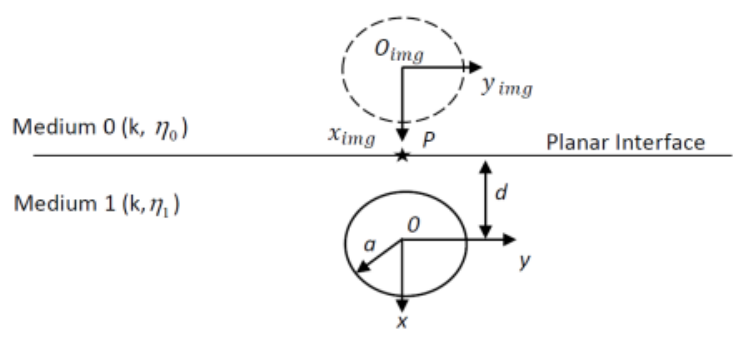

Figure 2: Buried PEMC circular cylinder in the lower halfspace and its image in the upper half-space.

Imposing the electric and magnetic field boundary conditions at the point $P$ on the interface (see Figure 2) corresponding to $\rho=\rho_{\text {imag }}=d, \phi=\pi$, and $\phi_{\text {imag }}=0$, given by

$$
\begin{aligned}
& E_{z}^{s, 0}+E_{z}^{r, 0}=E_{z}^{t, 0} \\
& H_{\phi}^{s, 0}+H_{\phi}^{r, 0}=H_{\phi}^{t, 0} \\
& E_{\phi}^{s, 0}+E_{\phi}^{r, 0}=E_{\phi}^{t, 0} \\
& H_{z}^{s, 0}+H_{z}^{r, 0}=H_{z}^{t, 0}
\end{aligned}
$$

yields the unknown coefficients. Substituting from (11)-(14) and (18)-(25) in (26)-(29), and considering the orthogonality of the exponential functions, we obtain

$$
\begin{gathered}
G_{n}^{0}=\left(\frac{2}{x_{n}}-1\right) B_{n}^{0} e^{j n \pi} \\
D_{n}^{0}=\frac{2 B_{n}^{0}}{x_{n}}
\end{gathered}
$$

with

$$
x_{n}=\left(\frac{\eta_{1}}{\eta_{0}}+1\right)
$$

$$
\begin{gathered}
Q_{n}^{0}=\left(\frac{2}{x_{n}}-1\right) C_{n}^{0} e^{j n \pi} \\
F_{n}^{0}=\frac{2 C_{n}^{0}}{x_{n}} .
\end{gathered}
$$

The propagating reflected fields which correspond to their components given in (22) - (25), upon reaching the buried PEMC cylinder, act as secondary incident fields and generate higher order scattered fields.

According to Graf's addition theorem, these reflected field components that act as secondary incident field components can be expressed as

$$
\begin{aligned}
& E_{z}^{r, 0}=\sum_{n=-\infty}^{\infty} \bar{G}_{n}^{0} J_{n}(k \rho) e^{j n \phi} \\
& E_{\phi}^{r, 0}=\sum_{n=-\infty}^{\infty} \bar{Q}_{n}^{0} J_{n}^{\prime}(k \rho) e^{j n \phi}
\end{aligned}
$$

where

$$
\begin{aligned}
\bar{G}_{n}^{0} & =\sum_{m=-\infty}^{\infty} G_{m}^{0} H_{m-n}^{(2)}(2 k d) \\
\bar{Q}_{n}^{0} & =\sum_{m=-\infty}^{\infty} Q_{m}^{0} H_{m-n}^{(2)}(2 k d) .
\end{aligned}
$$

The corresponding magnetic field components can then be obtained using Maxwell's equations.

In response to the secondary fields incident on the buried cylinder, the components of the fields scattered by it can be obtained from $(11)-(14)$, by replacing the superscript 0 by 1. The unknown coefficients with superscript 1 are then obtained by imposing the PEMC boundary condition at the surface of the PEMC cylinder. The resulting equations can be written as

$$
\left[\begin{array}{l}
B_{n}^{1} \\
C_{n}^{1}
\end{array}\right]=\left[\begin{array}{ll}
\aleph_{11, n} & \aleph_{12, n} \\
\aleph_{21, n} & \aleph_{22, n}
\end{array}\right]\left[\begin{array}{l}
\bar{G}_{n}^{0} \\
\bar{Q}_{n}^{0}
\end{array}\right]
$$

with $\aleph_{11, n}$ and $\aleph_{21, n}$ obtained from (15) and (16), respectively, when $A_{n}=-1 . \aleph_{12, n}=-\aleph_{21, n}$ and $\aleph_{22, n}$ can be obtained from $\aleph_{11, n}$ by replacing its functions with the derivatives with respect to the argument, and vice versa.

Using the coefficients in the left-hand side of (39) in (30), (31), (33), (34), (37), and (38) after altering the superscripts of the coefficients in these equations from 0 to 1 , the coefficients with superscript 1 on the left-hand side of these six equations can be evaluated. Repeating this sequence allows calculation of other unknown expansion coefficients with superscripts 2,3 , 4 , etc.

The components of the total electric field in the half-space above the planar interface are given by

$$
\begin{gathered}
\tilde{E}_{z}^{r}=E_{z}^{r}+\sum_{k} E_{z}^{t, k} \\
\tilde{E}_{\phi}^{r}=\sum_{k} E_{\phi}^{t, k} .
\end{gathered}
$$

The co-polar and cross polar reflection coefficients can then be written as

and 


$$
\begin{gathered}
\Gamma_{c p}=\frac{\tilde{E}_{z}^{r}}{E_{z}^{i}} \\
\Gamma_{x p}=\frac{\tilde{E}_{\phi}^{r}}{E_{z}^{i}} .
\end{gathered}
$$

\section{Numerical Results}

Results are presented to show how the co-polar and cross polar reflection coefficient magnitudes at the point $P$ in Fig. 1, vary with the size of the buried cylinder, for PEMC cylinders of different PEMC admittances, of different burial depths, and for different impedance ratios of the two isorefractive media. For convenience, all the normalized PEMC admittances have been expressed in the dimensionless form $M \eta_{1}=\tan v$.

To validate the formulation and the results obtained for a buried PEMC cylinder, we compare the results obtained using this formulation for $v=90^{\circ}$ and $v=0^{\circ}$, with the corresponding results obtained for buried perfect electric conducting (PEC) cylinders and perfect magnetic conducting (PMC) cylinders, respectively. For the cases of PEC and PMC cylinders, there are no cross-polar field components. Therefore, the expansion coefficients of the initial co-polar scattered field components are given by $B_{n}=J_{n}(k a) A_{n} / H_{n}^{(2)}(k a)$ for the PEC cylinder and by $B_{n}=J_{n}^{\prime}(k a) A_{n} / H_{n}^{(2) \prime}(k a)$ for the PMC cylinder. In Fig. 3 we show comparisons of $\left|\Gamma_{\mathrm{cp}}\right|$ at $P$ for a circular cylinder buried in an isorefractive medium at a depth of $2.5 \mathrm{~cm}$ from the planar interface, when $\eta_{0} / \eta_{1}=0.4$ and the electrical radius $k a$ of the cylinder varies from 0.05 to 1.6. Each of the cylinders is excited by an electric line source located at $3.0 \mathrm{~cm}$ from the interface. As can be seen, the results are in good agreement, verifying the validity of the formulation and the software used to obtain the results.

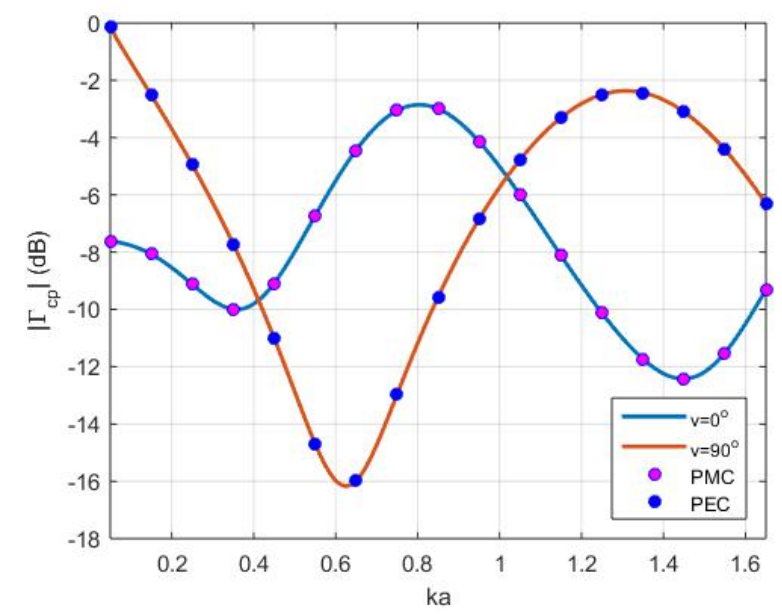

Figure 3: Comparing the variations of reflection coefficient magnitudes with $k a$ for buried PEMC circular cylinders of radius $a$, with $d=2.5 \mathrm{~cm}, \rho_{0}=3.0 \mathrm{~cm}$, and $\eta_{0} / \eta_{1}=0.4$, for $\nu=0^{\circ}$ and $v=90^{\circ}$, with those for equivalent PMC and PEC cylinders.

Figure 4 shows the variations of $\left|\Gamma_{\mathrm{cp}}\right|$ and $\left|\Gamma_{\mathrm{xp}}\right|$ at $P$ for a PEMC circular cylinder buried in an isorefractive medium at a depth of $2.5 \mathrm{~cm}$ from the planar interface, for five different values of $M \eta_{1}$, when $\eta_{0} / \eta_{1}=0.5$ and the electrical radius $k a$ of the cylinder varies from 0.05 to 1.6 . The electric line source creating the excitation is located in the isorefractive medium above the planar interface at a distance of $3.0 \mathrm{~cm}$ from it, and is of unit amplitude. In Fig. 3(a), the minima shift to the right and their sharpness increases with increasing $v$. In Fig. 4(b), we see that the curves for $v=15^{\circ}$ and $v=75^{\circ}$ are essentially the same and so are those for $v=30^{\circ}$ and $v=60^{\circ}$. Also, variations of $\left|\Gamma_{\mathrm{cp}}\right|$ with $k a$ are much rapid, when they are compared with the corresponding variations of $\left|\Gamma_{\mathrm{xp}}\right|$.

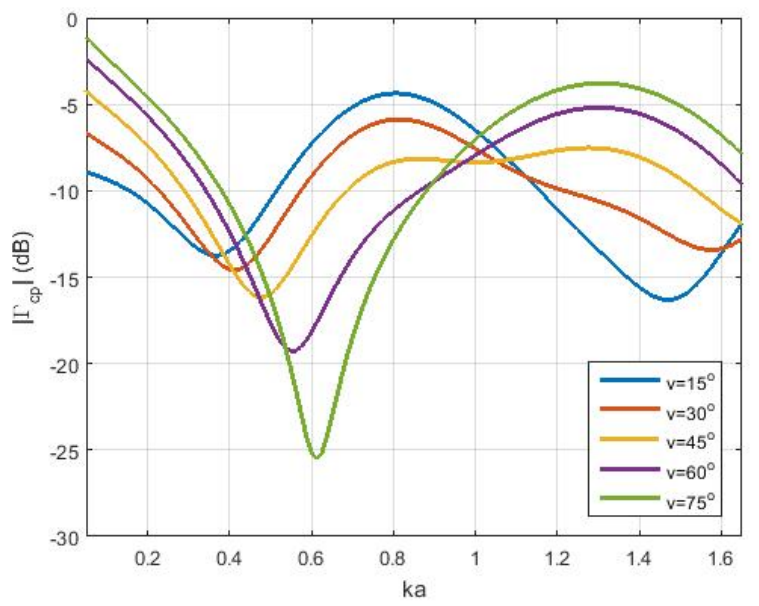

(a)

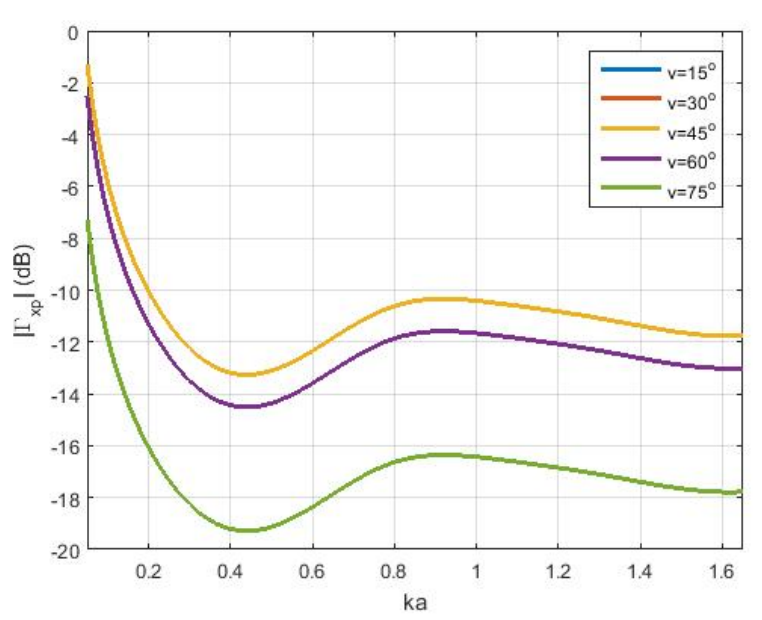

(b)

Figure 4: Variations of the reflection coefficient magnitudes with $k a$ for buried PEMC circular cylinders of radius $a$, with $d=2.5 \mathrm{~cm}, \rho_{0}=3.0 \mathrm{~cm}$, and $\eta_{0} / \eta_{1}=0.5$, for five different values of $v$; (a) co-polar coefficient; (b) cross polar coefficient.

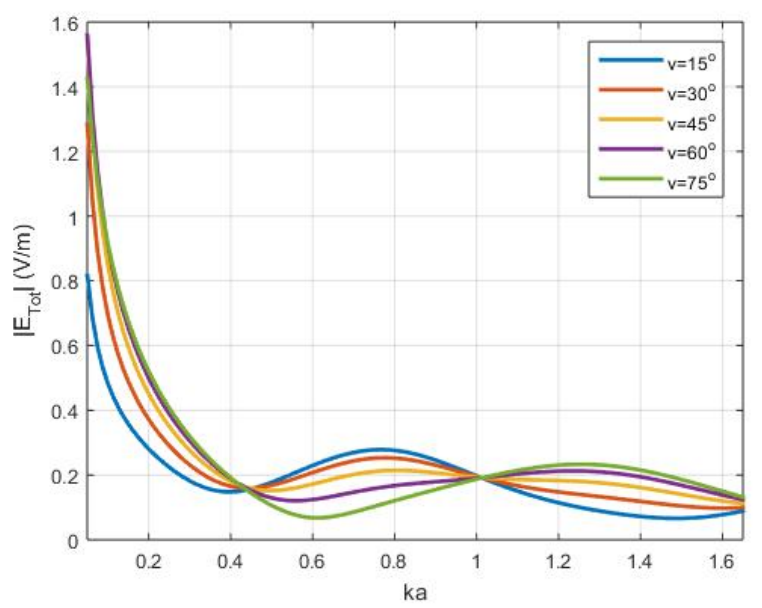


Figure 5: Variations of the total electric field magnitudes at $P$ with $k a$ for the buried cylinders of radius $a$ in Fig. 4 .

Variations of the total electric field magnitudes at $P$ with $k a$ for the buried cylinders of radius $a$ in Fig. 4 are shown in Fig. 5. Here we observe that the field magnitudes are rather high for smaller cylinders. Also, for $0.45 \leq k a \leq 1.0$, the magnitudes of the fields decrease as $v$ increases, whereas for other values of $k a$, the field magnitudes increase with $v$.

Figure 6 shows the variations of $\left|\Gamma_{\mathrm{cp}}\right|$ and $\left|\Gamma_{\mathrm{xp}}\right|$ at $P$ for the setup considered in Fig. 4, for 5 different impedance ratios of the isorefractive media defined by $y_{r}=\eta_{1} / \eta_{0}$, when the PEMC admittance of the buried cylinder is given by $M \eta_{1}=1$, and its electrical radius varies from 0.05 to 1.6 . As $y_{r}$ increases from 2.0 to 6.0 a similar sort of variation can be seen in the curves corresponding to both Figs. 6(a) and 6(b). But in Fig. 6(a), the magnitudes of the curves for a particular $k a$ increase as $y_{r}$ increases, whereas in Fig. 6(b), they decrease.

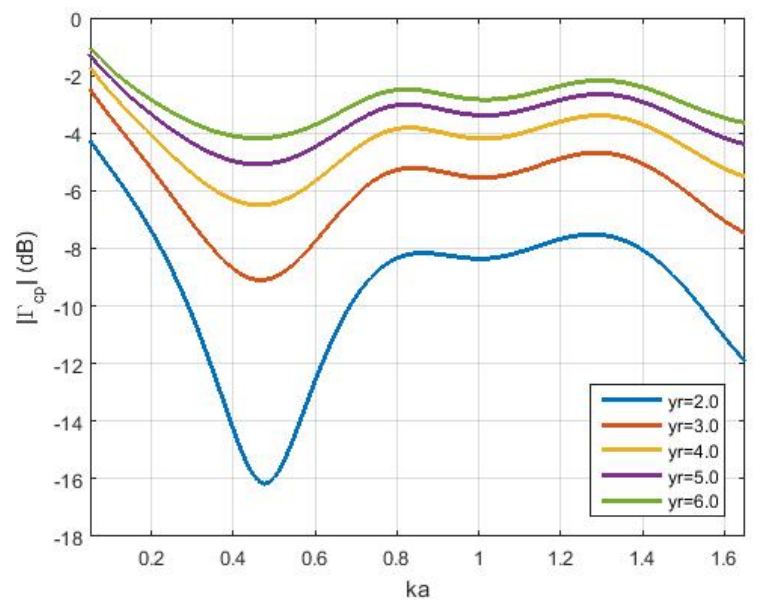

(a)

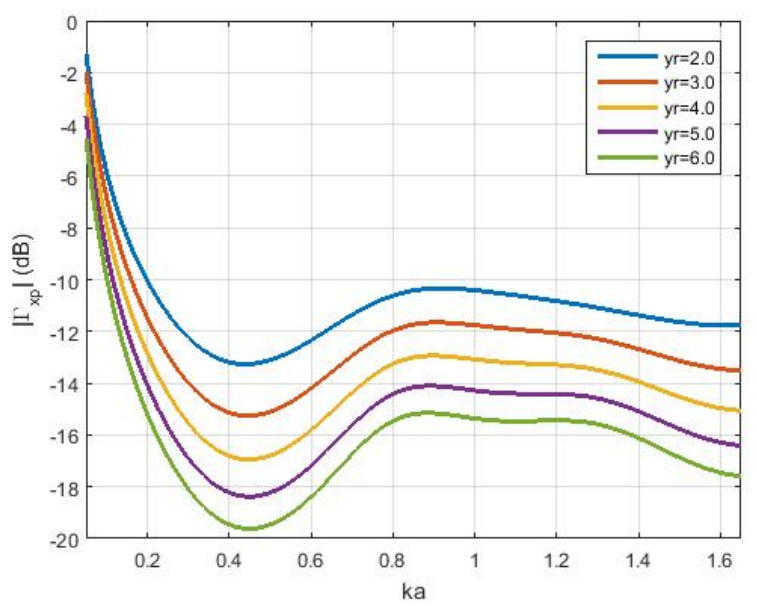

(b)

Figure 6: Variations of the reflection coefficient magnitudes with $k a$ for buried PEMC circular cylinders of radius $a$, with $d=2.5 \mathrm{~cm}, \rho_{0}=3.0 \mathrm{~cm}$, and $v=45^{\circ}$, for five different values of $y_{r}$; (a) co-polar coefficient; (b) cross polar coefficient.

Figure 7 shows the variations of $\left|\Gamma_{\mathrm{cp}}\right|$ and $\left|\Gamma_{\mathrm{xp}}\right|$ at $P$ for the setup considered in Fig. 4, for 5 different burial depths $d$ of the cylinder, when the PEMC admittance of the buried cylinder is given by $v=75^{\circ}$, and when its electrical radius varies from 0.05 to 1.6. As $d$ increases from 2.0 to 2.8 , the minima in Fig. 7(a) shift to the left, and their magnitudes increase. Also, variations of $\left|\Gamma_{\mathrm{cp}}\right|$ with $\mathrm{ka}$ in Fig. 7(a) are much rapid, when they are compared with the corresponding variations of $\left|\Gamma_{\mathrm{xp}}\right|$ in Fig. 7(b), and $\left|\Gamma_{\mathrm{xp}}\right|$ in Fig. 7(b) for a particular $k a$ decreases steadily, as the burial depth $d$ increases.

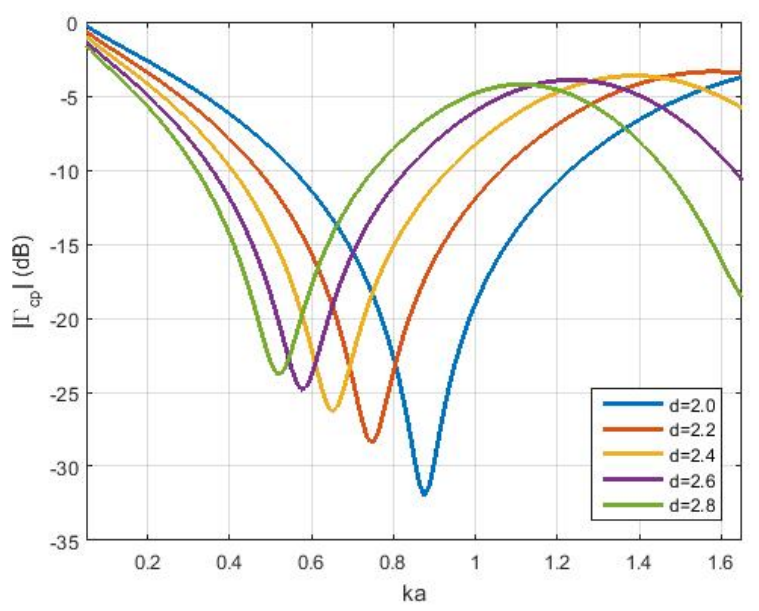

(a)

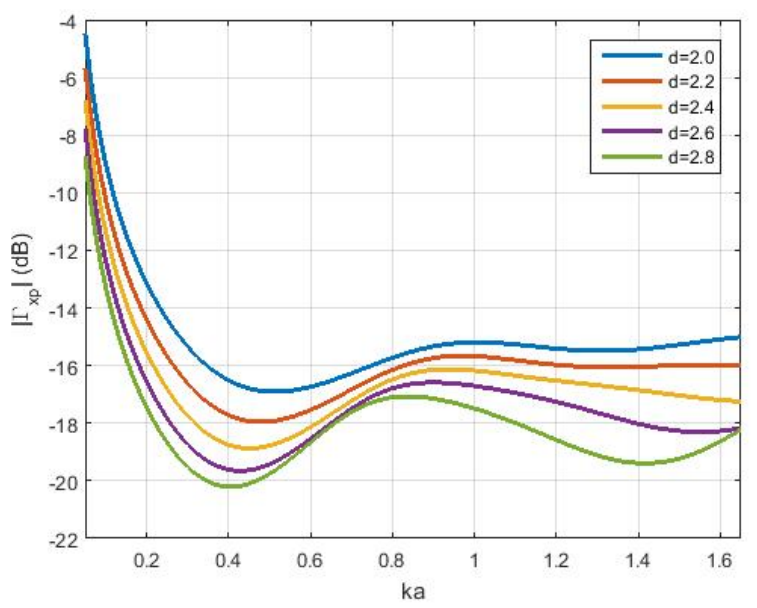

(b)

Figure 7: Variations of the reflection coefficient magnitudes with $k a$ for buried PEMC circular cylinders of radius $a$, with $\rho_{0}=3.0 \mathrm{~cm}, \eta_{0} / \eta_{1}=0.5$, and $v=75^{\circ}$, for five different values of the burial depth $d$; (a) co-polar coefficient; (b) cross polar coefficient.

\section{Conclusions}

A rigorous solution that is based on the multiple reflection of waves has been obtained for scattering by a PEMC circular cylinder buried in a half-space separated by a planar interface, when it is illuminated by an infinite electric line source located in the other half-space, with the half-spaces being isorefractive to each other. The presented solution is efficient, as it does not require inversion of matrices. The obtained numerical results are portrayed in the form of co-polar and cross polar reflection coefficient magnitudes, to exhibit how 
they vary with the size, and PEMC admittance of the buried cylinder, with the burial depth, and also with the impedance ratio of the isorefractive media. Moreover, results for analogous PEC and PMC buried cylinders can be obtained from this analysis, as special cases. The solution process could also be used for solving related inverse scattering problems.

\section{Acknowledgement}

Prof. A.-K. Hamid would like to acknowledge the help provided by the University of Sharjah, Sharjah, UAE.

\section{References}

[1] D. Erricolo and P. L. E. Uslenghi, "Exact radiation and scattering for an elliptic metal cylinder at the interface between isorefractive half space," IEEE Trans. Antennas Propag., vol. 52, no. 9, pp. 2214-2225, Sep. 2004.

[2] A. N. Askarpour and P. L. E. Uslenghi, "Exact radiation from dipole antennas on prolate spheroids coated with isorefractive and anti-isorefractive layers," IEEE Trans. Antennas Propag., vol. 60, no. 4, pp. 2129-2133, Apr. 2012.

[3] A. N. Askarpour and P. L. E. Uslenghi, "Exact radiation from dipole antennas on oblate spheroids coated with isorefractive and anti-isorefractive layers," IEEE Trans. Antennas Propag., vol. 60, no. 11, pp. 5476-5479, Nov. 2012.

[4] J. Zhang and X. He, "Asymptotic diffraction of a PEC cylinder located at a plane interface between isorefractive half-spaces," IEEE Trans. Antennas Propag., vol. 45, no. 6, pp. 3282-3287, Jun. 2017.

[5] S. F. Mahmoud, S. M. Ali, and J. R. Wait, "Electromagnetic scattering from a buried cylindrical inhomogeneity inside a lossy earth," Radio Sci., vol. 16, no. 6, pp. 1285-1298, Nov.Dec. 1981.

[6] B. P. D'Yakonov, “The diffraction of electromagnetic waves by a circular cylinder in a homogeneous half space," Bull. Acad. Sci., U.S.S.R.., Ser., vol. 9, pp. 950-955, 1959.

[7] A. Q. Howard, Jr., "The electromagnetic fields of a subterranean cylinder inhomogeneity excited by a line source, Geophysics, vol. 37, no. 6, pp. 975-984, Jun. 1972.

[8] S. O. Ogunade, "Electromagnetic response of an embedded cylinder for line current excitation," Geophysics, vol. 46, no. 1, pp. 45-52, Jan. 1981.

[9] Q. A. Naqvi and A. A. Rizvi, "Scattering from a cylindrical object buried in a geometry with parallel plane interfaces," PIER, vol. 27, pp. 19-35, 2000.

[10] M. Di Vico, F. Frezza, L. Pajewski, and G. Schettini, "Scattering by a finite set of perfectly conducting cylinders buried in a dielectric half-space: a spectral-domain solution," IEEE Trans. Antennas Propag., vol. 53, no. 2, pp. 719-727, Feb. 2005.

[11] F. Frezza, L. Pajewski, C. Ponti, G. Schettini, and N. Tedeschi, "Electromagnetic scattering by a metallic cylinder buried in a lossy medium with the cylindrical wave approach," IEEE Geosci. Remote Sens. Lett., vol. 9, pp. 179-183, 2012.

[12] F. Frezza, L. Pajewski, C. Ponti, and G. Schettini, "Line source scattering by buried perfectly conducting circular cylinders," Int. J. Antennas Propag., vol. 2012, 7 pages, 2012.

[13] M. Nasr, E. Hashish, and I. Eshrah, "Fast analytical evaluation of EM scattered fields due to buried cylinders," in Proc. IEEE AP-S Int. Symp., 2013, Lake Buena Vista, FL, USA, Jul. 7-13, 2013.
[14] M. A. Nasr, I. A. Eshrah, and E. A. Hashish, "Electromagnetic scattering from a buried cylinder using a multiple reflection approach: TM case," IEEE Trans. Antennas Propag., vol. 62, no. 5, pp. 2702-2707, May 2014.

[15] A.-K. Hamid and F. Cooray, "Scattering by buried PEMC cylinder using iterative procedure", in Proc. $18^{\text {th }}$ Mediterranean Microwave Symposium (MMS2018), Istanbul, Turkey, 31 Oct.-3 Nov., 2018.

[16] A.-K. Hamid and F. Cooray, "Scattering from a buried PEMC cylinder illuminated by a normally incident plane wave propagating in free space", Advanced Electromagnetics Journal, vol. 8, pp. 1-7, 2019.

[17] I. Diamandi and J. N. Sahalos, "Frequency and time domain analysis of the scattered field of buried dielectric targets," Archiv fur Electrotechnik, vol. 77, pp. 441-449, Sep. 1991.

[18] J. L. Izadian, L. Peters, Jr., and J. H. Richmond, "Computation of scattering from penetrable cylinders with improved numerical efficiency," IEEE Trans. Geosci. Remote Sens., vol. GE-22, no. 1, pp. 52-61, Jan. 1984.

[19] Y. Z. Umul, "Scattering of electromagnetic waves by a perfect electromagnetic conductor half-screen," Optik, vol. 181, pp. 383-388, Mar. 2019.

[20] R. Ruppin, "Scattering of electromagnetic radiation by a perfect electromagnetic conductor cylinder," J. Electromagn. Waves Appl., vol. 20, pp. 1853-1860, 2006. 